\title{
Tin disulfide segregation on CZTS films sulfurized at high pressure
}

\author{
Alejandro Alvarez ${ }^{\mathrm{a}}$, Stephen Exarhos ${ }^{\mathrm{a}}$, Lorenzo Mangolini ${ }^{\mathrm{a}, \mathrm{b}^{*}}$
}

a) Mechanical Engineering Department, University of California Riverside, 900 University Avenue, Riverside, CA 92521, USA. E-mail: Imangolini@engr.ucr.edu

b) Materials Science and Engineering Program, University of California Riverside, 900 University Avenue, Riverside, CA 92521, USA

\section{Abstract}

CZTS thin films were synthesized by annealing of $\mathrm{Zn}, \mathrm{Cu}$, and $\mathrm{Sn}$ stacked layers under sulfur atmosphere. Pressure was controlled by varying the mass of the sulfur charges introduced into the closed annealing chamber. Films sulfurized at higher pressure show clear segregation of hexagonal $\mathrm{SnS}_{2}$, suggesting that $\mathrm{Sn}$ reacts with gaseous $\mathrm{S}$ to form a solid-state phase, leading to an inhomogeneous 3D film morphology. Lower pressure synthesis yields uniform layers mainly composed of CZTS. Variations in film morphology suggest that different reaction pathways take place as the pressure is raised. Formation of gaseous SnS is favored at lower pressure, while nucleation of solid $\mathrm{SnS}_{2}$ preferentially occurs at higher pressure. Influence of the cooling rate after annealing was also studied. We have found that this affects the film stoichiometry, while the film morphology did not show relevant dependence on this parameter.

Keywords: CZTS; sulfurization pressure; thin films; physical vapor deposition; photovoltaics

\section{Introduction}

$\mathrm{Cu}_{2} \mathrm{ZnSnS}_{4}$ is a promising material for thin film photovoltaics. CZTS is composed of earth abundant, non-toxic elements, making it an ideal candidate for the scalable and economically feasible production of thin film solar cells [1-3]. However, efficiency records lower than those achieved by the commercially available CIGS technology have kept CZTS production away from industrial levels $[4,5]$. Among the various CZTS synthesis processes, sulfurization of stacked layers of metallic precursors has become attractive due to its controllable stoichiometry and use of a clean, non-toxic approach [6-8]. Within this particular technique, the influence of 
several parameters such as annealing temperature, annealing time, utilized substrate, preheating, and precursor stoichiometry have been thoroughly studied [9-14]. However, there are only a few studies on sulfurization pressure. The lack of information addressing this topic leads to inconsistencies associated to the role of pressure in grain size and post-annealing stoichiometry $[15,16]$. Additionally, procedures related to the sulfurization steps vary widely. It is therefore unreliable to reach unequivocal conclusions from results that follow different sulfurization routes. A better understanding of the chemical pathways through which CZTS is synthesized is crucial in order to identify the source of efficiency problems mainly related to low open circuit voltage and short recombination times [17]. In this manuscript we present a direct and concise analysis on the influence that sulfurization pressure has on CZTS in a closed system.

\section{Materials and methods}

$50 \mathrm{~nm}$ of $\mathrm{Zn}$ were initially deposited on a quartz substrate by RF magnetron sputtering, followed by the deposition of $80 \mathrm{~nm}$ of $\mathrm{Cu}$ and $110 \mathrm{~nm}$ of $\mathrm{Sn}$ by electron beam evaporation. This combination of techniques facilitates the deposition of a smooth-surface film for each metallic precursor. Five samples were independently annealed in a sealed quartz ampule $\left(203 \mathrm{~mm}\right.$ long, $7 \mathrm{~mm}$ in diameter) at $500^{\circ} \mathrm{C}$ for $8 \mathrm{~h}$. A base pressure of $1 \times 10^{-5}$ Torr was achieved with an Oerlikon Leybold TURBOVAC SL 80 turbo pump. The ampules were heated in a tube furnace at a rate of $7^{\circ} \mathrm{C} / \mathrm{min}$. After annealing, the films were let to cool down naturally for 120 minutes. One end of the ampule was gently pushed out of the heating zone in order to induce constant sulfur condensation in that region and away from the sample. The sulfurization pressure was controlled by modifying the amount of sulfur introduced into the closed system. Pressure calculations were based on the application of the ideal gas law. In accordance to previous reports, the mole fractions of the various molecular species present in sulfur vapor $\left(S_{8}\right.$ to $\left.S_{2}\right)$ were considered for these estimations [18]. The corresponding pressure data for each mass value, listed in increasing order, is approximately 32 (1mg), 95 (3mg), 191 (6mg), 287 (9mg), and 383 (12mg) Torr. SEM/EDS analysis was performed on a FEI Nova NanoSEM 450 system equipped with an Oxford Instruments Aztec Synergy software. EDS data was obtained from diverse areas of each sample. The average stoichiometry and standard deviation were recorded. XRD 
characterization was held on a PANalytical Empyrean X-ray system using CuKa radiation with a wavelength of $1.74 \mathrm{~nm}$. A Horiba LabRam HR instrument, equipped with a 532nm laser source, was used for Raman analysis.

\section{Results}

Fig. 1 shows low and high magnification SEM images of the CZTS films sulfurized at different pressures. There are no obvious morphological differences among the samples synthesized at lower pressure (Fig. 1a1f). On the other hand, three-dimensional structures are visible in the films sulfurized at 287 and 383 Torr

(Fig. 1g-1j). These features are randomly dispersed over the surface of the films. The regions in between the 3D structures are very similar to those shown in the previous samples.

\begin{tabular}{|c|c|c|c|c|c|c|c|c|c|c|c|}
\hline \multirow{2}{*}{\multicolumn{4}{|c|}{ at\% Before sulfurization }} & \multirow{3}{*}{$\begin{array}{c}\text { Pressure } \\
\text { (Torr) } \\
-\end{array}$} & \multicolumn{7}{|c|}{ at\% After sulfurization } \\
\hline & & & & & \multicolumn{7}{|c|}{ Natural cooling (120 minutes) } \\
\hline Sample & $\%$ Zn & $\% \mathrm{Cu}$ & $\%$ Sn & & $\% Z n$ & $\% \mathrm{Cu}$ & $\%$ Sn & $\% \mathrm{~S}$ & $\mathrm{Cu} / \mathrm{Sn}$ & $\mathrm{Zn} / \mathrm{Sn}$ & $\mathrm{Cu} / \mathrm{Zn}$ \\
\hline 1 & $24.4 \pm 0.4$ & $46.5 \pm 0.5$ & $29.1 \pm 0.5$ & 32 & $16.7 \pm 1.5$ & $22.7 \pm 0.8$ & $14.4 \pm 1.2$ & $46.2 \pm 0.7$ & 1.58 & 1.16 & 1.36 \\
\hline 2 & $23.8 \pm 0.4$ & $47.0 \pm 0.5$ & $29.1 \pm 0.1$ & 95 & $15.4 \pm 1.5$ & $24.0 \pm 1.0$ & $14.5 \pm 1.0$ & $46.1 \pm 1.0$ & 1.65 & 1.06 & 1.56 \\
\hline 3 & $24.4 \pm 0.3$ & $46.6 \pm 0.2$ & $29.0 \pm 0.3$ & 191 & $16.2 \pm 1.9$ & $23.9 \pm 0.8$ & $14.1 \pm 1.4$ & $45.8 \pm 1.4$ & 1.70 & 1.15 & 1.48 \\
\hline 4-SA1 & $25.2 \pm 0.9$ & $46.1 \pm 1.0$ & $28.6 \pm 0.6$ & 287 & $18.1 \pm 0.9$ & $24.4 \pm 0.8$ & $12.8 \pm 0.6$ & $44.6 \pm 1.2$ & 1.90 & 1.41 & 1.35 \\
\hline 4-SA2 & $25.2 \pm 0.9$ & $46.1 \pm 1.0$ & $28.6 \pm 0.6$ & 287 & $16.0 \pm 0.4$ & $23.7 \pm 0.6$ & $14.4 \pm 0.5$ & $45.8 \pm 0.5$ & 1.64 & 1.11 & 1.48 \\
\hline 5-SA1 & $23.7 \pm 0.6$ & $46.6 \pm 0.3$ & $29.6 \pm 0.6$ & 383 & $17.8 \pm 0.3$ & $25.2 \pm 0.4$ & $12.7 \pm 0.4$ & $44.3 \pm 0.5$ & 1.99 & 1.41 & 1.41 \\
\hline \multirow[t]{2}{*}{ 5-SA2 } & $23.7 \pm 0.6$ & $46.6 \pm 0.3$ & $29.6 \pm 0.6$ & 383 & $15.1 \pm 0.3$ & $23.2 \pm 0.4$ & $15.3 \pm 1.1$ & $46.5 \pm 0.8$ & 1.54 & 0.99 & 1.54 \\
\hline & & & & & \multicolumn{7}{|c|}{ Slow cooling (240 minutes) } \\
\hline 6 & $24.4 \pm 0.4$ & $46.5 \pm 0.5$ & $29.1 \pm 0.5$ & 32 & $11.8 \pm 1.0$ & $20.9 \pm 1.0$ & $16.2 \pm 0.9$ & $51.0 \pm 1.1$ & 1.29 & 0.73 & 1.77 \\
\hline 7 & $25.2 \pm 0.9$ & $46.1 \pm 1.0$ & $28.6 \pm 0.6$ & 287 & $12.6 \pm 0.5$ & $21.5 \pm 1.0$ & $15.7 \pm 0.7$ & $50.2 \pm 0.8$ & 1.37 & 0.80 & 1.71 \\
\hline
\end{tabular}

Table 1. Summary of EDS analysis performed in all samples reported in the present manuscript.

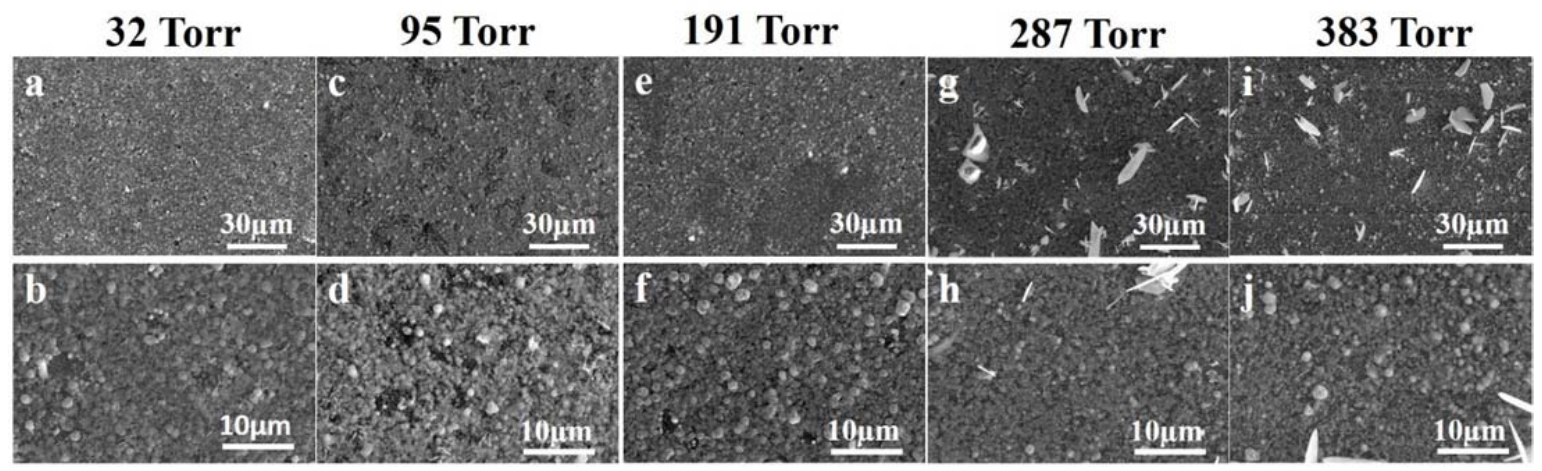


EDS analysis is broken down in Table 1. Due to film inhomogeneity, data for samples 4 and 5 is reported under Scanning Areas 1 and 2 (SA1, SA2). SA2 includes every relevant feature in the sample, whereas SA1 does not comprise the characteristic 3D morphologies solely present in the films synthesized at higher pressures. Fig. 2a-2c show a graphic representation of the stoichiometry values reported in Table 1 while only considering data from SA1. Cu/Sn and Zn/Sn ratios (Fig. 2a and 2b, respectively) are considerably larger at 287 and 383 Torr. The Cu/Zn ratio (Fig. 2c), however, does not show a clear dependence on the sulfurization pressure. This information suggests that there is major Sn loss in SA1 as the pressure is raised. When including the 3D structures into these measurements (i.e., reporting values from SA2) the Cu/Sn, and $\mathrm{Zn} / \mathrm{Sn}$ ratios (Fig. $2 \mathrm{~d}$ and $2 \mathrm{e}$, respectively) are remarkably similar. There is therefore no material loss at higher pressures, but rather a displacement of Sn into clearly defined regions of the film. Additional EDS mapping of these 3D features is displayed in Fig. 2g-2k. Strong S and Sn signals are detected in the region of interest (Fig. $2 \mathrm{j}$ and $2 \mathrm{k}$, respectively). In contrast, $\mathrm{Cu}$ and $\mathrm{Zn}$ signals at Fig. $2 \mathrm{~h}$ and $2 \mathrm{i}$ are much weaker in that particular sector.
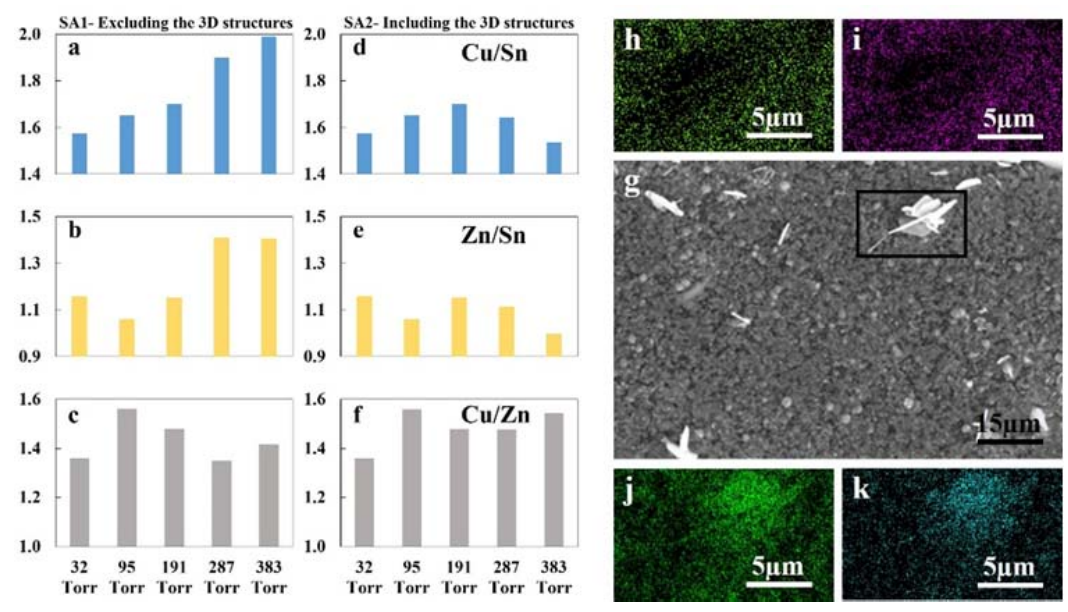

XRD data from the as-synthesized films is shown in Fig. 3a. Peaks associated to $\mathrm{CZTS}_{2} \mathrm{Cu}_{2} \mathrm{SnS}_{3}$, and $\mathrm{ZnS}$ are present at $28.5^{\circ}, 33.1^{\circ}, 47.5^{\circ}$, and $56.2^{\circ}$. Additional weak peaks at $34.6^{\circ}, 50.0^{\circ}$, and $52.1^{\circ}$ indicate there is minor segregation of secondary phases in all films. These reflections may be related to the $\mathrm{Cu}_{39} \mathrm{~S}_{28}$ alloy (JCPD 036 0380) [19]. Peaks from $\mathrm{SnS}_{2}$ at $15.0^{\circ}$ and $32.1^{\circ}$ become visible at 287 and 383 Torr, adding up to 
the evidence of $\mathrm{SnS}_{2}$ segregation at high pressures. The remarkable similarity among the CZTS, ZnS, and $\mathrm{Cu}_{2} \mathrm{SnS}_{3}$ XRD peaks makes it impossible to fully confirm the presence of CZTS by solely employing this characterization technique [20]. Raman analysis is a useful complimentary tool for CZTS detection. Fig. 3b shows the Raman spectrum for each of the five samples synthesized at various sulfurization pressures. Strong CZTS peaks at $337 \mathrm{~cm}^{-1}$ and $289 \mathrm{~cm}^{-1}$ are readily detected in all the films. As pressure increases, a hexagonal $\mathrm{SnS}_{2}$ peak at $314 \mathrm{~cm}^{-1}$ becomes stronger. This trend is consistent with the EDS and XRD data presented above.
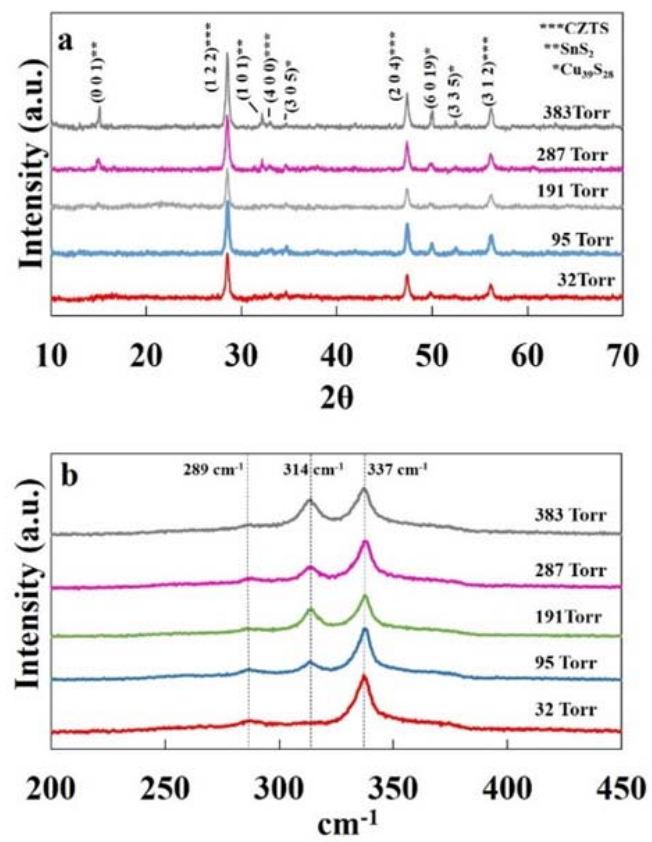

\section{Discussion}

Previous investigations on CZTS sulfurization in closed systems emphasize the importance solid-gas phase reactions have in the chemical pathways that lead to the formation of CZTS crystals [21]. At low sulfurization pressure, the proposed mechanism consists in the formation of gaseous SnS as a result of the interaction between solid Sn with the S atmosphere. Further incorporation of SnS into the film leads to a solid-state interaction with CuS phases to form $\mathrm{Cu}_{2} \mathrm{SnS}_{3}$. Finally, $\mathrm{ZnS}$ diffuses into $\mathrm{Cu}_{2} \mathrm{SnS}_{3}$ to yield CZTS. The presence of $\mathrm{SnS}_{2}$ at high pressure suggests that the chemical route explained above is partially altered under these 
anomalous conditions. Instead of forming SnS (g), interaction between Sn (s) and S (g) favors the growth of solid $\mathrm{SnS}_{2}$ three-dimensional structures. Since these features appear in the final film morphology, it is evident that there is no further reaction between $\mathrm{SnS}_{2}$ and other phases during the sulfurization process. Higher stability of $\mathrm{SnS}_{2}(\mathrm{~s})$ over $\mathrm{SnS}(\mathrm{g})$ may readily explain this phenomenon.

An additional experiment was designed in order to discard the effects that the cooling rate may have over the film morphology. Two extra samples, sulfurized at 32 and 287 Torr, underwent a slower cooling process. The overall cooling time was increased from 120 to 240 minutes. Stoichiometry values as obtained from EDS measurements are reported in Table 1 (samples 6 and 7). A larger amount of Sn and S is detected. Zn and Cu losses are also evident. Nonetheless, despite the stoichiometric variations there were no morphological changes associated to the cooling rate. Film features are very similar to those shown in Fig. 1a-1b and $1 \mathrm{~g}-1 \mathrm{~h}$, suggesting that there is no influence of the cooling rate in the formation of $\mathrm{SnS}_{2} 3 \mathrm{D}$ structures. It is apparent that the cooling rate is a relevant and often overlooked factor in the final stoichiometry of the film. Gaseous sulfur left in the ampule keeps reacting with the sample while cooling. These notable stoichiometric variations shed light on the great complexity related to the formation mechanisms of CZTS, in which subtle changes in any parameter play a substantial role on the final film properties.

\section{Conclusions}

The effects of pressure during CZTS sulfurization in a closed system was investigated. Higher pressure yields samples with evident segregation of $3 \mathrm{D} \mathrm{SnS}_{2}$ structures randomly dispersed along the film. Collected data suggests that the formation of these structures follows different reaction pathways to those reported for lower pressure processes. A greater chemical stability of solid $\mathrm{SnS}_{2}$ compared to that of gaseous $\mathrm{SnS}$ explains the fact that the formation of $\mathrm{SnS}_{2}$ does not lead to further interaction with other phases in the film. Additionally, the cooling rate has a substantial influence on the sample final stoichiometry. However, the surface morphology is unaffected by changes in this parameter.

\section{Acknowledgements}


The authors acknowledge the support of the National Science Foundation under award number 1351386

(CAREER) and of the "Consejo Nacional de Ciencia y Tecnología" (CONACYT, Mexico). Raman and XRD were performed at the Analytical Chemistry Instrumentation Facility (ACIF) at UC Riverside. SEM characterization was performed at the Central Facility for Advanced Microscopy and Microanalysis (CFAMM) at UC Riverside.

\section{References}

[1] B. A. Andersson, Materials availability for large-scale thin-film photovoltaics, Prog. Photovoltaics Res. Appl. 8 (2000) 61-76.

[2] D.B. Mitzi, O. Gunawan, T.K. Todorov, K. Wang, S. Guha, The path towards a high-performance solution-processed kesterite solar cell, Sol. Energy Mater. Sol. Cells. 95 (2011) 1421-1436.

[3] K. Ito, T. Nakazawa, Electrical and Optical Properties of Stannite-Type Quarternary Semiconductor Thin Films, Jpn. J. Appl. Phys. 27 (1988) 2094-2097.

[4] W. Wang, M.T. Winkler, O. Gunawan, T. Gokmen, T.K. Todorov, Y. Zhu, et al., Device characteristics of CZTSSe thin-film solar cells with $12.6 \%$ efficiency, Adv. Energy Mater. 4 (2014) 1-5.

[5] M.A. Green, E. Keith, Y. Hishikawa, W. Warta, E.D. Dunlop, Solar cell efficiency tables (Version 45), Prog. Photovoltaics Res. Appl. 23 (2015) 1-9.

[6] T.P. Dhakal, C. Peng, R. Reid Tobias, R. Dasharathy, C.R. Westgate, Characterization of a CZTS thin film solar cell grown by sputtering method, Sol. Energy. 100 (2014) 23-30.

[7] A. Emrani, T.P. Dhakal, C. Peng, C.R. Westgate, CZTS solar cells fabricated by fast sulfurization of sputtered Sn/Zn/Cu precursors under H 2 S atmosphere, leee Pvsc 40. (2014) 2-4.

[8] J. Zhang, B. Long, S. Cheng, W. Zhang, Effects of sulfurization temperature on properties of CZTS films by vacuum evaporation and sulfurization method, Int. J. Photoenergy. 2013 (2013) 1-7.

[9] A.-J. Cheng, M. Manno, a. Khare, C. Leighton, S. a. Campbell, E.S. Aydil, Imaging and phase identification of Cu2ZnSnS4 thin films using confocal Raman spectroscopy, J. Vac. Sci. Technol. A Vacuum, Surfaces, Film. 29 (2011) 051203.

[10] S. Exarhos, K.N. Bozhilov, L. Mangolini, Spray pyrolysis of CZTS nanoplatelets, Chem. Commun. 50 (2014) 11366-11369.

[11] P. A. Fernandes, P.M.P. Salomé, a. F. Sartori, J. Malaquias, A. F. da Cunha, B.A. Schubert, et al., Effects of sulphurization time on Cu2ZnSnS4 absorbers and thin films solar cells obtained from metallic precursors, Sol. Energy Mater. Sol. Cells. 115 (2013) 157-165.

[12] H. Guan, H. Shen, C. Gao, X. He, Sulfurization time effects on the growth of Cu2ZnSnS4 thin films by solution method, J. Mater. Sci. Mater. Electron. 24 (2013) 2667-2671. 
[13] Y. Lin, S. Ikeda, W. Septina, Y. Kawasaki, T. Harada, M. Matsumura, Mechanistic aspects of preheating effects of electrodeposited metallic precursors on structural and photovoltaic properties of Cu2Zn SnS4 thin films, Sol. Energy Mater. Sol. Cells. 120 (2014) 218-225.

[14] C. Malerba, F. Biccari, C.L. Azanza Ricardo, M. Valentini, R. Chierchia, M. Müller, et al., CZTS stoichiometry effects on the band gap energy, J. Alloys Compd. 582 (2014) 528-534.

[15] J. He, L. Sun, Y. Chen, J. Jiang, P. Yang, J. Chu, Influence of sulfurization pressure on Cu2ZnSnS4 thin films and solar cells prepared by sulfurization of metallic precursors, J. Power Sources. 273 (2015) 600-607.

[16] K. Zhang, Z. Su, L. Zhao, C. Yan, F. Liu, H. Cui, et al., Improving the conversion efficiency of Cu2ZnSnS4 solar cell by low pressure sulfurization, Appl. Phys. Lett. 104 (2014) 141101.

[17] A. Polizzotti, I.L. Repins, R. Noufi, S.-H. Wei, D.B. Mitzi, The state and future prospects of kesterite photovoltaics, Energy Environ. Sci. 6 (2013) 3171-3182.

[18] H. Rau, T.R.N. Kutty, J.R.F. Guedes De Carvalho, Thermodynamics of sulphur vapour, J. Chem. Thermodyn. 5 (1973) 833-844.

[19] C. Wang, Z. Fang, F. Fan, X. Dong, Y. Peng, S. Hao, et al., Facile synthesis of size-tunable Cu39S28 micro/nano-crystals and small-sized configuration enhanced visible-light photocatalytic activity, CrystEngComm. 15 (2013) 5792.

[20] P. a. Fernandes, P.M.P. Salomé, a. F. da Cunha, Study of polycrystalline Cu2ZnSnS4 films by Raman scattering, J. Alloys Compd. 509 (2011) 7600-7606.

[21] M.C. Johnson, C. Wrasman, X. Zhang, M. Manno, C. Leighton, E.S. Aydil, Self-Regulation of Cu/Sn Ratio in the Synthesis of Cu 2 ZnSnS 4 Films, Chem. Mater. 27 (2015) 2507-2514.

\section{Captions}

Fig. 1. Low and high magnification SEM images showing the surface morphology of the CZTS samples sulfurized at various pressures.

Fig. 2. $\mathrm{Cu} / \mathrm{Sn}(\mathrm{a}, \mathrm{d}), \mathrm{Zn} / \mathrm{Sn}(\mathrm{b}, \mathrm{e})$, and $\mathrm{Cu} / \mathrm{Zn}(\mathrm{c}, \mathrm{f})$ stoichiometry ratios of samples sulfurized at various pressures. Data from both SA1 and SA2 is included for the samples synthesized at higher pressures. (h-k) EDS mapping of the framed region in (g). S (j) and $\mathrm{Sn}(\mathrm{k})$ comprise most of the elemental stoichiometry of that specific feature.

Fig. 3. (a) XRD data showing characteristic CZTS peaks and weak $\mathrm{Cu}_{39} \mathrm{~S}_{28}$ reflections in all films. Hexagonal $\mathrm{SnS}_{2}$ reflections are observed at 287 and 383 Torr. (b) Raman analysis of samples sulfurized under various 
pressure conditions. A strong CZTS signal is observed in all films. A hexagonal $\mathrm{SnS}_{2}$ peak becomes more visible as pressure increases. 